\title{
THE PATHOLOGY OF EMPHYSEMA
}

\author{
J. Gough, M.D., F.C. Path. \\ Welsh National School of Medicine, Cardiff.
}

IN THIs Cardiff Symposium of the Postgraduate Medical Journal, I should first explain why the Pathology Department of the Welsh National School of Medicine became interested in emphysema as one of its main subjects for research. It arose out of a study of thousands of respiratory cripples in the coal mining industry. In the early part of this century coal miners were a healthy population apart from accidents, but by the third decade it was evident that respiratory disease of serious proportions was arising. At first it was thought that this was silicosis (Cummins and Sladden, 1930), affecting those miners who were exposed to rock dust as well as to coal. It soon became evident, however, that although there were cases of silicosis in those who worked especially on rock, there was a far higher incidence of respiratory troubles in miners who worked on the coal seams and in coal trimmers who load coal into ships. The name "coal worker's pneumoconiosis" was applied and is now accepted in law. A study of the lungs of miners showed that there are two quite separate forms of pneumoconiosis (Gough, 1947). The one is a massive fibrosis while the other consists of discrete nodules of coal with comparatively little fibrosis but which subsequently become surrounded by a characteristic emphysema now known as "focal dust emphysema". A technique was evolved to demonstrate this emphysema more effectively. This is the whole lung paper section method devised by Gough and Wentworth $(1957 / 58 ; 1960)$. It is carried out on lungs fixed in expansion. The large section method was next used for a study of respiratory diseases of the general population and it revealed (Gough, 1952) two fundamentally different forms of emphysema. In the one there was a selective involvement of the centres of the lung lobules. This is known as central or centrilobular emphysema. In the other form there was no selection of the kind of air spaces dilated and since this lesion was not based on the lobule but often affected the greater part of both lungs it was referred to

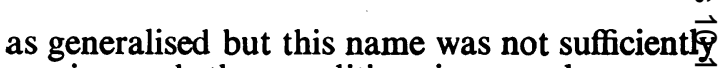
precise and the condition is now known as "panacinar" or "panlobular". In view of the common use of the terms lobule and acinus the classification of emphysema, it is necessat to define them. Anatomy
The Lobule (also called the secondary lobuts
of Miller)
The lung lobule is the smallest component which is bounded by fibrous tissue septa. This is the definition given by the Basle Convention and is used by anatomists, and is an important anatomical structure for reference. The lobuldeg are $1-2 \mathrm{~cm}$. in diameter. Their outlines are 80 ơ easily seen in some parts of the lungs but those of us who have studied coal miners' lungs are very familiar with the lobular structure because in many miners the fibrous tissue septa becomo impregnated with coal (Fig. 1). The lobuleg tend to be pyramidal with their apices directed towards the hilum. They have their own ai and blood supply and lymphatic drainage. They are virtually miniature lungs, a feature whict becomes important in the correlation of emphysema with disturbed function because uneven functional capacity in different units of parts of the lung is readily brought out by. function tests and a study of the patholog 8 shows that some lobules may be severele affected while neighbouring ones may be virtually normal.

\section{The Acinus}

It is much more difficult to define the acinus because different authors have used this term in different ways. In classifying the differento types of emphysema a Ciba Foundatiog Symposium (1959) used 'acinus' for the unit of respiratory tissue distal to the terminat bronchiole. All the components in the acinus so defined bear alveoli. This acinus consists of respiratory bronchioles, alveolar ductsp alveolar sacs and alveoli. The Ciba Symposiun recommended that the classification of emphy 


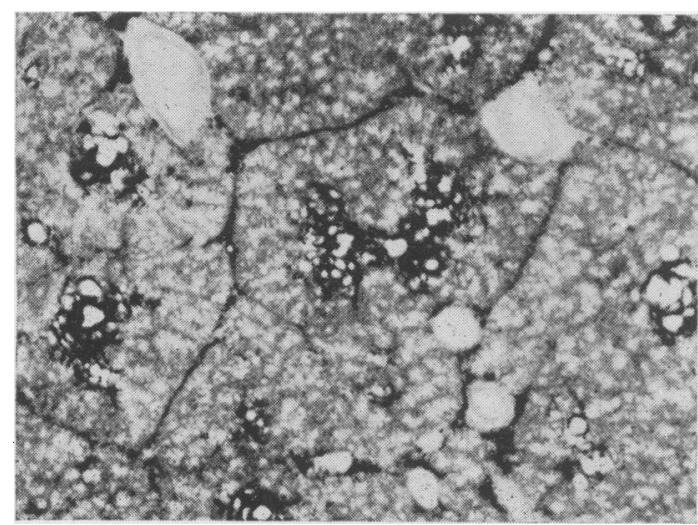

Fig. 1.-Lobules outlined by dust in the connective tissue septa. In the centres of the lobules is an early stage of focal dust emphysema. Air spaces at the periphery of the lobules are intact. x2.3.

sema should be based on pathological anatomy because respiratory function tests and radiology are not sufficiently reliable to warrant a precise classification at the present time. Function tests do not distinguish the different varieties of chronic non-specific obstructive lung diseases, emphysema, bronchitis and asthma with sufficient precision: improvements in this respect, however, are developing and combined radiological, functional and anatomical classifications are appearing. It will be necessary, however, to study large series of cases where there is correlation of functional disturbance in life with the pathological anatomy before these classifications are acceptable. Limiting the definition and classification, therefore, strictly to pathology, the Ciba Symposium proposed the following: -

"Emphysema is a condition of the lung characterized by increase beyond the normal in the size of air spaces distal to the terminal bronchiole either from dilatation or from destruction of their walls."

Emphysema can be diagnosed and classified consistently only on preparations from lungs distended and fixed before they are cut. The simplest technique is intrabronchial infusion of fixative. In some cases identification of the anatomical origin of enlarged spaces may require such techniques as the study of serial sections, or stereoscopic microscopy of lung slices.

The term emphysema is restricted to dilatation of air spaces distal to the terminal bronchiole in order to differentiate emphysema from dilatations which include structures proximal to the terminal bronchiole, as in various forms of honeycomb lung.
1. Unselective distribution beyond the terminal bronchiole (panacinar emphysema)

(a) Dilatation alone (e.g. compensatory $\stackrel{\frac{1}{2}}{2}$ emphysema and emphysema due to $C$ partial main bronchus obstruction).

(b) Destruction of the walls of air spaces (panacinar destructive emphysema).

2. Selective distribution beyond the terminal bronchiole.

(i) Predominantly affecting respiratory bronchioles.

(a) Dilatation alone (e.g. focal emphysema due to dust).

(b) Destruction of the walls of air spaces (centrilobular emphysema).

(ii) Predominantly affecting alveolar ducts $\exists$ and sacs.

(a) dilatation alone.

(b) destruction.

3. Irregular distribution beyond the terminal bronchiole (irregular emphysema).

Of the five pathologists who took part in the symposium one, Dr. L. McA. Reid, proposed an alternative arrangement of the various types of emphysema but all the pathologists were agreed what the types are. The difference o $\$$ opinion was whether the precise anatomicaI location should be the primary basis of the classification, or whether destruction should be the primary criterion. The following alternative arrangement of the classification was proposed: -

1. Dilatation alone.

(a) Unselective distribution (compensatory emphysema and emphysema due to partial main bronchus obstruction).

(b) Selective distribution predominantly affecting respiratory bronchioles (e.g. focal emphysema due to dust).

2. Destruction of the walls of air spaces.

(a) Unselective distribution (panacinar destructive emphysema).

(b) Selective distribution predominantly affecting respiratory bronchioles (centrilobular emphysema).

(c) Irregular distribution (irregular emphysema).

\section{Centrilobular Emphysema (Gough, 1952;} Leopold and Gough, 1957)

This is a common condition in the general population. The centrilobular origin of emphysematous spaces is best observed in lobules which are not severely diseased. The changes are destructive even in the smallest lesions, and the ragged air spaces at the centre contrast with the normal pattern of lung tissue. A 


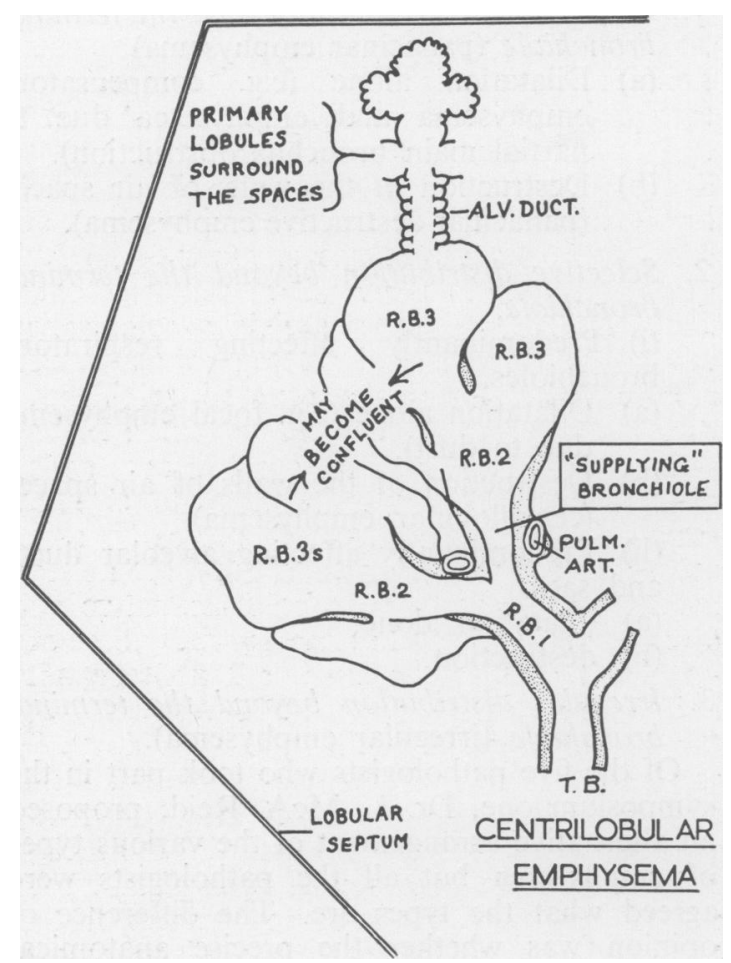

Fig. 2.-Diagram of a lobule showing the characteristics of centrilobular emphysema.

T.B.-Terminal bronchiole.

R.B. 1, 2 \& 3-Three orders of respiratory bronchioles. x3.5 approximately.

mulberry-like cluster of emphysematous spaces develops (Fig. 2). The spaces become confluent as they enlarge. Carbon in amounts common in the lungs of town-dwellers is collected around the spaces and in their partitions, thus helping to delineate the lesion but dust is not an essential component. The emphysematous cuuster is marked off into loculi by partitions which do not, however, form complete separations. These partitions radiate from one bronchiole which provides the airway to the cluster. This bronchiole is called the "supplying" bronchiole. Patent communications between the bronchioles and the emphysematous spaces are traceable in all lesions although the calibre of these passages varies. The "supplying" bronchioles show histological changes of chronic inflammation and the inflammation having extended peripherally, centrilobular emphysema is one of the results due to breakage of the walls of respiratory bronchioles. Such peripheral extension of inflammation may occur very irregularly in the lung so that unaffected lobules are contiguous with lobules showing centrilobular emphysema. Spread of inflamz mation also leads to breakage of the partitions radiating from respiratory bronchioles. In the normal lung these partitions help keep ope the bronchioles to which they are attached Rupture of the partitions causes the bronchioleg to collapse during expiration thus producing: air trapping.

\section{Panacinar (syn. panlobular emphysemå Generalized emphysema)}

The panacinar type is conveniently classified into sub-groups. One appears to be an atrophige process of unknown cause and this is called primary. The other is commonly associated with chronic bronchitis. Centrilobular and panacinar emphysema often occur together in lungs showing chronic bronchitis.

\section{(a) Primary type}

In this type the various air-containing space in the acini enlarge, i.e. it is unselective an affects respiratory bronchioles, alveolar ducts, alveolar sacs and alveoli. Not only does the lesion appear uniformly in the individual lobules but it is a condition which often affect 8 large part of both lungs. Commonly the whole of both lungs show the condition. It is for reason that I preferred originally to calf "generalized". It is a common disease in the general population; not infrequently it is a incidental finding and may be an exaggeration of the ageing process, because from childhoog up to adult life the air spaces show a natural increase in size. One suspects, however, that it may not be a simple ageing process because many old people, especially older women, show no sign of it. This difference in the sex incidence would seem to suggest that the emphysematous changes seen in old people's lungs are moge associated with wear and tear and the effects of disease rather than the mere passage of time. It should be pointed out, however, that there are senile changes in the lungs which lead reduced respiratory capacity but which are net emphysema. These changes are thought to related to the physico-chemical changes in the connective tissues. (See Ageing of the Lurg, Ed. Cander, L., and Moyer, J. H., 1964).

(b) Panacinar emphysema associated wi⿺h chronic bronchitis

Some of the most severe cases of chronic non-specific obstructive lung disease consist of chronic bronchitis and panacinar emphyseroa (Fig. 3). In these cases the inflammatoxy changes extend to the smallest bronchioles aid 


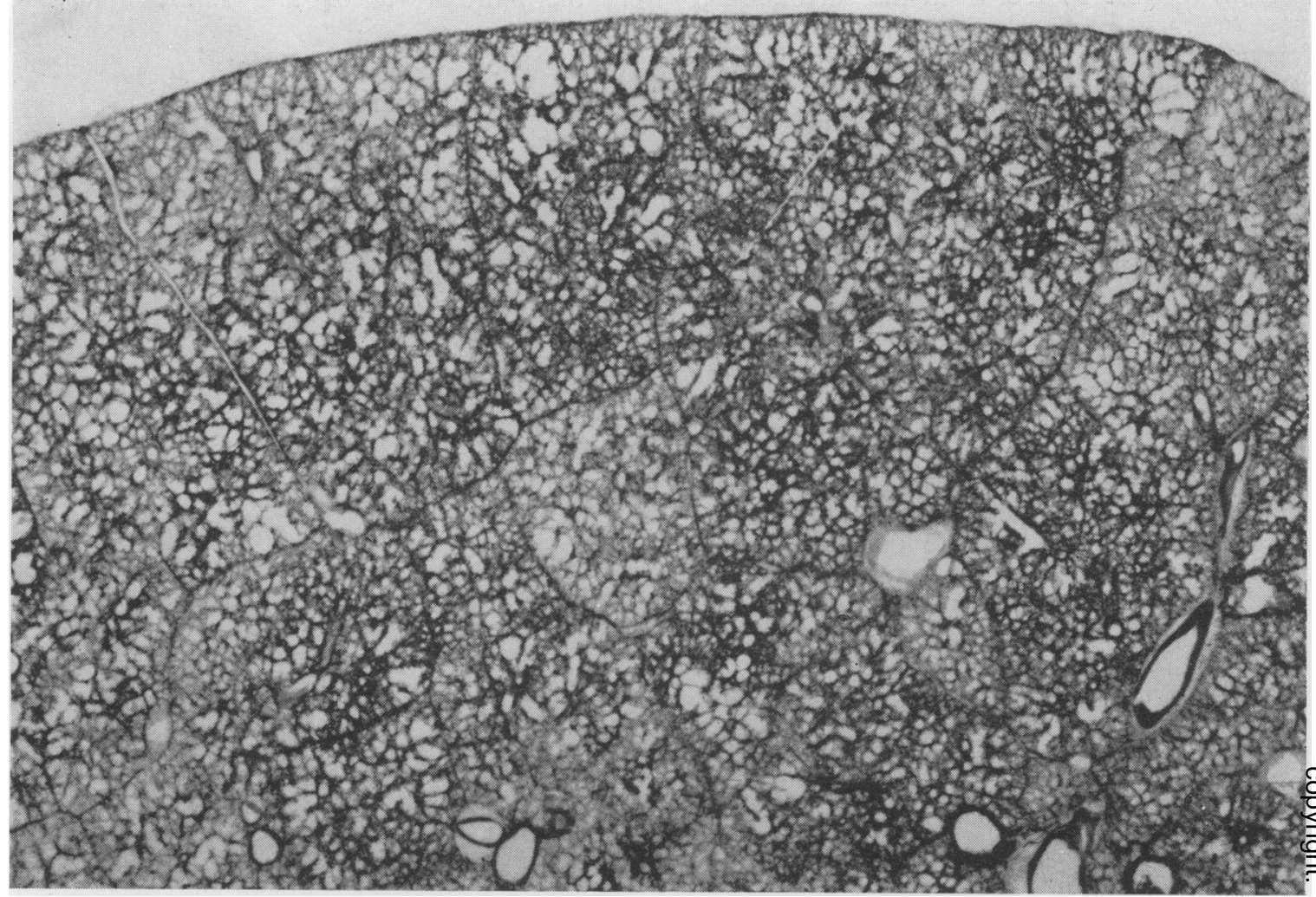

FIG. 3.-Panacinar (panlobular) emphysema. The outline of the lobules are faint but distinguishable. $\mathrm{x} 1.6$.

into the respiratory part of the lungs so that many of the alveolar walls are thickened by inflammatory cells and rupture of these walls occurs. Destructive panacinar emphysema may occur alone but is often accompanied by the centrilobular type and in our fatal cases of cor pulmonale we find this combination much more common than destructive panacinar alone.

\section{(c) Unilateral and unilobar panacinar em- physema}

Emphysema of a panacinar kind may be restricted to one lung or to one lobe. There are two forms. One is neonatal, the newly born child becoming severely dyspnoeic: air is trapped in one lobe due to a defect in the supplying bronchus. This lobe continues to expand and compresses the remainder of the lung and the contralateral lung. The disease constitutes a surgical emergency. The defect found in the bronchus in most cases is deficiency of the bronchial cartilages so that the bronchus is flaccid and collapses and acts as a valve Other defects, however, such as flap-like folds in the bronchial wall occur and give rise to a valve action. The second type of unilateral or unilobar emphysema is discovered by routine radiography, the affected part being hyperlucent. These cases may be found in childhood or in adult life. They are thought to be the result of childhood infections rather than maldevelopment, but in some of the cases that I have seen the bronchi have been extremely flaccid possibly due to developmental defect. In other cases, however, there has been evidence of severe chronic bronchiolitis. A good discussion on a case of this disease is in Brit. med. J., $1965, \mathbf{i}, 708$.

\section{Focal dust emphysema}

(a) Occupational

Simple pneumoconiosis of coal workers is a focal disease. It is seen as black spots throughout the lungs from microscopic size up to $5 \mathrm{~mm}$. in diameter. These may be soft and 
indistinguishable by feel from the surrounding lung or may be hard and felt as nodules, depending on the amount of fibrosis. In relation to both the soft and the hard foci the air spaces become dilated up to 1 to $5 \mathrm{~mm}$. diameter (Fig. 1). These black areas of emphysema constitute the characteristic feature of the disease. Focal dust emphysema is caused by atrophy of the smooth muscle in the walls of the respiratory bronchioles which are ensheathed in dust. There is also greater inspiratory pull on the bronchioles because of consolidation of the vesicular tissues by dust (Heppleston, 1954). When seen in large sections focal dust emphysema is characteristically widespread and in cases of severe disease, involves a high proportion of all the lobules. In the affected lobules one or several blackened areas of emphysema are seen. Central parts of the lobules are more affected than the tissue adjacent to the interlobular septa. The disease starts as a dilatation of respiratory bronchioles (Heppleston, 1954) but destructive changes develop in the later stages and completely disorganize the structure within the lobules. Emphysema has been long recognized as occurring around silicotic nodules, but it appears especially pronounced in the pneumoconiosis due to coal and other inert dusts.

\section{(b) Non-occupational-soot emphysema}

It is necessary to distinguish centrilobular emphysema due to bronchiolitis from focal dust emphysema which can occur in the general population exposed to atmospheres heavily polluted with smoke and soot. This soot emphysema is a lesser form of the focal dust emphysema found in dusty occupations such as coal mining. It may be due to associated pollutants especially $\mathrm{SO}_{2}$ and $\mathrm{NO}_{2}$ rather than the dust itself. Confusion has arisen in descriptions because the two forms of emphysema, centrilobular emphysema and focal dust emphysema, commence in centres of lobules. The importance of distinguishing them is, however, that the emphysema due to the dust has less effect upon the respiratory function than the centrilobular emphysema due to bronchiolitis. Soot emphysema in the general population is usually without functional disturbance but severe dyspnoea and cor pulmonale are common with bronchitic centrilobular emphysema. The differences are related to the initial destructive character of the centrilobular lesion while focal dust emphysema commences with a simple dilatation of the air spaces and destruction occurs only in the more advanced and severe industrial cases.

\section{Irregular Emphysema}

In the Ciba classification the term "irregular章 is used for the kind of emphysema found ie the neighbourhood of scars. This is ver黑 common especially in the upper parts of the lungs in relation to healed areas of tuberculosis but may arise from many inflammatory cons ditions which lead to fibrosis. Contraction of the fibrous tissue is regarded as the most important cause of the emphysema but it is highly probable that inflammatory destructiog is also a contributory cause. Sometimes these localised emphysemas become bullous in type the supplying bronchi being partially obstructed. Such bullae may have little disturbance of lung function but, on the other hand, air trapping may be so marked that there 想 pressure on the surrounding lung.

\section{Practical Application of the Ciba Classification}

Pathologists have tried the practical use of the Ciba classification and in the majority of instances it is suitable provided that the luoggs have been fixed in expansion and examined by such techniques as the whole lung pape mounted sections of Gough and Wentwort or the barium impregnation method of Hearg (1958). Fixation by formalin fume has als been successfully used in the study of emphysema. Most of the types will fall in the categories described above but in certain instances the emphysema is so severe and destructive in character that it is not possible to say how it originated, i.e. whether it cons menced as centrilobular or panacinar, or some other way, so that a class of "indete? minate" is necessary. Both centrilobular and panacinar emphysemas may proceed to the complete disintegration of the structure within the lobule. One can speculate on the likely nature in a particular case if the rest of the lung is not so severely affected and if it shops one or both the basic emphysema types. The American Thoracic Society (1962) ha adopted the Ciba classification to a large extefy but they have limited the definition to include destructive changes only and did not regard simple over-expansion as a form of emphysema. This leaves the pathologist in a quandary arfad he would need to use another term such as the classical one "pulmonum volumen auctum I think most pathologists would prefer to reta\$n over-distention as a form of emphysema rather 
than separate it under the title "pulmonary over-inflation" which is what the Americans propose.

\section{Relative Incidence of Types of Emphysema}

In a series of 140 examples of emphysema from the general population which Leopold and I (1957) examined post mortem, we found that in 65 there was panacinar emphysema alone whereas in the remaining 75 there was centrilobular in addition to panacinar emphysema.

Thurlbeck (1963) examined 150 lungs at the Harvard Medical School Department of Pathology. They were from patients who had died at the Massachusetts General Hospital, Boston. 138 of the specimens had been from unselected autopsies and 21 were of "severe anatomic pulmonary emphysema". The lungs had been fixed in expansion. He found centrilobular emphysema, panacinar emphysema, a destructive lesion commencing in the alveolar ducts which he regarded as a variant or a precursor of panacinar emphysema and the irregular or scar type of emphysema but no cases of focal dust emphysema. Thus he was able to differentiate the types of emphysema according to the Ciba classification. In the unselected cases centrilobular emphysema alone or dominant was present in $21.7 \%$; panacinar emphysema alone or dominant in $13.8 \%$. In the cases which were specially selected as severe emphysema, centrilobular was pure or dominant in 6 , panacinar pure or dominant in 10 , mixed 3 and unclassifiable 3.

In an investigation at the Hammersmith Hospital in London, Heard and Izukawa (1964) found in a series of 50 lungs from consecutive autopsies, no evidence of any gross difference in the patterns and incidence of emphysema between London and our findings in Cardiff. Centrilobular emphysema is commoner in these two capitals than in Boston. Heard was careful to distinguish the focal dust type of emphysema associated with deposition of London dust. In order to distinguish this he referred to it as "distensive", pointing out that it is similar to the focal emphysema in coal workers.

\section{Emphysema and Cardiorespiratory Function}

\section{The relation of emphysema to cor pulmonale}

When a series of cases of chronic non-specific obstructive pulmonary disease is studied post mortem there is no consistent relationship between the severity of emphysema and the development of cor pulmonale. Extensive destructive emphysema means a proportional loss of the capillary bed but it is evident that the body can stand a very severe loss of this kind without necessarily developing cor pulmonale. In the series of cases mentioned above, Leopold and I found that of the 75 cases in which there was centrilobular as well as panacinar emphysema there was right ventricular hypertrophy in 41 whereas in 65 cases in which there was panacinar emphysema alone right heart hypertrophy was present in only nine. For this purpose we defined the right ventricular hypertrophy as a thickness of $6 \mathrm{~mm}$. or more. Recently we have preferred to use the weight of the right ventricle separated from the rest of the heart taking $70 \mathrm{~g}$. as normal. Of Heard and Izukawa's 50 cases in London, four had died of cor pulmonale and each of these had severe emphysema which consisted of both panacinar and centrilobular varieties and each of these four patients had chronic bronchitis. Wyatt, Fischer and Sweet (1962) found hypertrophy of the right ventricle frequently associated with generalised panacinar emphysema but they used the low figure of $49 \mathrm{~g}$. as the upper limit of the normal weight of this ventricle.

Of the chronic bronchitis-emphysema com plex in Britain it would seem that the bronchitis: or more accurately bronchiolitis is an important component in the development of cor pulmonale. Severe cases of chronic bronchitis $\stackrel{\circ}{\circ}$ and bronchiolitis without significant emphysema $\overrightarrow{\vec{P}}$ may die of cor pulmonale. Organic changes in the blood vessels are not present with sufficient consistency to be regarded as the major cause of the pulmonary hypertension. Functional disturbances, particularly hypoxia, have 3 to be invoked to explain the stress on the right heart. In cases of severe panacinar emphysema in which there is cor pulmonale I have fre- o quently found evidence of inflammation in the bronchioles extending into the alveolar walls, 웅 whereas the primary type of panacinar emphysema, atrophy of the alveolar walls and not inflammation, is the finding. In the inflam- NO matory type of panacinar emphysema several blocks of tissue need to be examined because $\widetilde{N}$ the inflammatory changes are not uniform $\mathbb{N}$ throughout the lung but related in patches to the bronchiolitis whereas in other parts the alveolar walls are thin.

\section{Emphysema and airways obstruction}

Emphysema is very commonly associated with airways obstruction but these two terms $\stackrel{\Phi}{\Phi}$ 
should not be taken as synonyms. There is now considerable evidence that airways obstruction can occur with little or no emphysema. This conclusion is based upon the finding of instances of severe chronic bronchitis with only very little destructive emphysema (Gough and Leopold, 1962). Such individuals may have been called emphysematous during life because they had marked airways obstruction. Simpson, Heard and Laws (1963) described three patients showing clinical and physiological features of chronic obstructive lung disease, though none showed radiological evidence of emphysema by established criteria. There was gross impairment of ventilatory function but an insignificant degree of emphysema was found at necropsy using special methods of examination. These observations were based on Heard's method of examining the lungs post mortem and they agree with my findings with paper mounted sections.

McNab, Grove and Nariman (1961) suggest that study of the effects of exercise on arterial oxygen saturation and on ventilation equivalent for oxygen may show whether anatomical emphysema is present or not. They found that centrilobular emphysema was associated with gross physiological defects. Bates and Christie (1964) conclude that in the correlation of structure and function in emphysema it is unfortunately true that the simplest ventilatory tests are of little value in this regard, but they feel that, in the majority of these patients, the combination of careful history-taking, detailed radiography and a complete assessment of function enables the physician to build up a fairly accurate picture of the probable morphological changes in the lungs.

\section{The Lungs in Fatal Status Asthmaticus}

Among the cases I studied of chronic lung disease in the general population there were some who had been diagnosed as asthma and had died in status asthmaticus. These had been seen by Dr. D. A. Williams during life and diagnosed by him as typical asthma. In most instances their lungs at autopsy did not show destructive emphysema. The lungs from the asthmatics were distended as is seen in drowning and they showed the classical appearances of plugging of the bronchi with dense mucus. Histologically there was infiltration with eosinophiles but, whereas in bronchitics there was much destruction of the walls of the air spaces, in the asthmatics the emphysema was mainly simple over-expansion.
Most of our fatal cases of asthma hape been in women. In 101 deaths from statess asthmaticus analysed by Dr. D. A. Williams, two-thirds were females and this distribution was found in all age groups. It might be arguẹd that the absence of destructive emphysema wgs mainly due to the fact that most of the patients were women but the same is true of some men who died in status asthmaticus. Williams and Leopold (1959) reported the autopsy findings of 25 adults, 19 females and six male, all of whom had died in status asthmaticus. The lungs were examined in my department. The average age of death was 56 years and têA were over 60 years. The average duration $8 \mathrm{ff}$ asthma was 21 years, three were over 40 years, yet in none of the female cases nor in foun out of six of the male cases was there ary destructive emphysema. Williams (1964) con siders that cigarette smoking accounts for thes higher incidence of destructive emphysema th male asthmatics.

Of course, individuals who started with asthma in childhood or later and subsequent庻 developed bronchitis had the pathological find ings of bronchitis. In localities where bronchitg is very common, as in London with its poll tided atmosphere, cases of uncomplicated ast may be rarer. With the clean air in Cardiff: do not have such difficulty in separatin "asthma" from chronic bronchitis. We ca?, however, only be sure of the post mortem diag nosis of asthma if death is due to status asthmaticus. If an asthmatic dies of a cause other than asthma there may be no evidence of asthmatic changes in the lungs.

\section{Experimental Emphysema}

Many attempts have been made to produce emphysema in experimental animals. Because of the view held for many years that the prime cause of emphysema is obstruction to the at flow most experiments have been based upon this view. Attempts at producing emphysema by partial obstruction have, however, has varying success. A summary of the earlier work is given by Rasmussen and Adams (1942). The禺 themselves failed to produce emphysema io dogs by over-distention simulating repeate asthmatic attacks extending over severg months. It is significant, however, that one of their dogs developed a spontaneous infection of the lungs and in it emphysema developeg and the authors remarked that "It is mos suggestive that the factor of infectio when added to repeated over-distention 
is significant in the production of pulmonary emphysema". Likewise Anderson and colleagues (1964) failed to produce emphysema in dogs by means of a Venturi valve in the trachea but when they combined this obstruction with the destructive effect of dilute nitric acid, they produced emphysema. Incidentally they found that "retired" greyhounds had a significant degree of natural emphysema. Wright and Kleinerman (1963) produced experimental centrilobular emphysema by exposing guinea pigs to nitrogen dioxide, a common constituent in polluted atmospheres. This same gas adsorbed on to carbon also had a focal destructive effect on the lungs of mice, (Boren, 1964). Centrilobular emphysema has been produced in rats by Gross, Babyak, Tolker, and Kaschak (1964) by the action of papain. These various investigations confirm our observation of the vulnerability of the respiratory bronchioles emphasizing the relationship of centrilobular emphysema to external agents which would include atmospheric pollutants as well as infections. Anderson and colleagues (1964) using paper-mounted sections, have found a close correlation between centrilobular emphysema and cigarette smoking but found no such relationship between panacinar emphysema and smoking.

\section{Summary}

For satisfactory post mortem study of emphysema the lungs should be expanded to a size approximating that present during life. This is best done by formalin either in solution or as a fume. The study is greatly enhanced if whole lung sections mounted on paper by the Gough and Wentworth technique, or impregnated with barium sulphate, by Heard's technique, is used. Two fundamentally different anatomical forms of emphysema are seen, centrilobular, a destructive lesion affecting primarily the respiratory bronchioles and panacinar (panlobular) in which all varieties of air spaces in the lobule expand. One form of the panacinar type consists of simple dilatation while the other is destructive. Focal dust emphysema is a specific variety seen most frequently in coal workers. It is also centrilobular in position and commences with dilatation of respiratory bronchioles but later may lead to destruction of the walls of the air spaces. A minor form of this disease occurs in the general population in the form of soot emphysema from polluted atmospheres.
The prevalent chronic non-specific obstructive pulmonary disease in Britain frequently causes 3 cor pulmonale. The commonest finding in such $\stackrel{\varnothing}{\circ}$ instances is a combination of chronic bronchitis $c$ (including bronchiolitis) and centrilobular and $\overrightarrow{\vec{S}}$ panacinar emphysema. In some instances the bronchitis is accompanied by only panacinar emphysema and in fewer instances bronchitis $\frac{\overline{0}}{\bar{n}}$ is present with insignificant emphysema. This $\widetilde{\nabla}$ emphasizes the importance of the bronchitic component as a cause of pulmonary hyper- $₫$ tension in the bronchitis-emphysema complex. $\vec{\circ}$ The cor pulmonale is apparently not solely due $\overrightarrow{\vec{H}}$ to reduction of the vascular bed and the $\vec{\omega}$ thickening of blood vessels, although these $\frac{8}{\circ}$ undoubtedly contribute, but also to functional disturbance, probably hypoxia. Preliminary experimental work in animals confirms the vulnerability to damage of the respiratory $\vec{v}$ bronchioles which has been observed in man. is Long standing primary spasmodic asthma, if $\mathrm{N}$ not complicated by bronchitis, does not usually $\frac{\text { ? }}{2}$ lead to destructive emphysema in non-smokers.

\section{REFERENCES}

Anderson, A. E., AzUCy, A., Batchelder, T. L., and FORAKER, A. G. (1964): Experimental Analysis in Dogs of the Relationship between Pulmonaz Emphysema Alveolitis and Hyperinflation, Thorase 19, 420.

Anderson, A. E., Hernandez, J. A., Eckert, P., and FORAKER, A. G. (1964): Emphysema in Lung Macrosections Correlated with Smoking Habits, Science, 144, 1025.

BATES, D. V., and CHRISTIE, R. V. (1964): Respiratory Function in Disease, p. 219, Philadelphia and London: W. B. Saunders.

BOREN, H. G. (1964): Carbon as a Carrier Mechanism for Irritant Gases, Arch. environm. Hlth., 8, 119.

CANDER, L., and MoYer, J. H. (1964): Ageing of the Lung. The Tenth Hahnemann Symposium, New York and London: Grune \& Stratton.

Cummins, S. L., and SladDen, A. F. (1930): Coal Miner's Lung: an Investigation into the Anthracotic Lungs of Coal Miners in South Wales, J. Path. Bact., 33, 1095.

AMERICAN THORACIC SOCIETY (1962): Definitions and 을 Classification of Chronic Bronchitis, Asthma and $D$ Pulmonary Emphysema. A Statement by the American Thoracic Society, Amer. Rev. resp. Dis., 85, 762.

GougH, J. (1947): Pneumoconiosis in Coal Workers in Wales, Occup. Med., 4, 86.

GougH, J. (1952): Discussion on the Diagnosis of

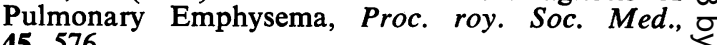
45, 576 .

Gough, J., and WenTwORTH, J. E. (1959): Correlation of Roentgenological and Pathological Changes in $\Phi$ Some Diseases of the Lung. Harvey Lectures $\stackrel{\mathscr{f}}{-}$ 1957/58. Series 53, 171. New York: Academic 0 Press.

Gough, J., and WeNTwORTH, J. E. (1960): Recent $\stackrel{\circ}{\mathbb{D}}$ Advances in Pathology, 7th Edition, London: J. \& A. Churchill. 
Gough, J., and LeOpold, J. G. (1962): Techniques used in the Study of Lung Pathology: The Anatomy of Emphysema. Ciba Foundation Symposium on Pulmonary Structure and Function, p. 280, London: J. \& A. Churchill.

Gross, P., BABYaK, M. A., TOlKER, E., and KaschaK, M. (1964): Enzymatically Produced Pulmonary Emphysema, J. occup. Med., 6, 481.

Heard, B. E. (1958): A Pathological Study of Emphysema of the Lungs with Chronic Bronchitis, Thorax, 13, 136

Heard, B. E., and Izukawa, T. (1964): Pulmonary Emphysema in 50 Consecutive Male Necropsies in London, J. Path. Bact., 88, 423.

HePpleston, A. G. (1954): The Pathogenesis of Simple Pneumokoniosis in Coal Workers, J. Path. Bact., 67, 51.

LEOPOLD, J. G., and Gough, J. (1957): The Centrilobular Form of Hypertrophic Emphysema and its Relation to Chronic Bronchitis, Thorax, 12, 219.

MCNab, G. R., Grove, W. S., and Nariman, S. (1961): A Comparison of Physiological and Pathological Findings in Chronic Bronchitis and Emphysema with Special Reference to Response to Exercise, Thorax, 16, 56.

Rasmussen, R. A., and Adams, W. E. (1942):
Experimental Production of Emphysema, Ar⿳⺈. intern. Med., 70, 379.

Simpson, T., Heard, B., and LAwS, J. W. (19638: Severe Irreversible Airways Obstruction withqu Emphysema, Thorax, 18, 361.

CiBA GUeST SYMPOSIUM (1959): Terminolog Definitions and Classification of Chronic Pulmonary Emphysema and Related Conditions. A Report \&f

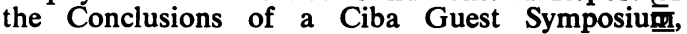
Thorax, 14, 286.

Thurlbeck, W. M. (1963): The Incidence Pulmonary Emphysema, Amer. Rev. resp. Da., 87, 206.

WilliamS, D. A., and Leopold, J. G. (1959): Death from Bronchial Asthma, Acta allerg. (Kbh.), 14, 83.

Williams, D. A. (1964): The Natural History of Asthma, 5th International Congress of Allergologg, p. 186, Madrid.

Wright, G. W., and Kleinerman, J. (1963): The J. Burns Amberson Lecture. A Consideration the Etiology of Emphysema in Terms of Contem porary Knowledge, Amer. Rev. resp. Dis., 88, 605 .

WyatT, J. P., Fischer, V. W., and Sweet, H. Z. (1962): Panlobular Emphysema: Anatomy and Pathodynamics, Dis. Chest, 41, 239. 